\title{
Poincaré on the Foundation of Geometry in the Understanding
}

Jeremy Shipley

\begin{abstract}
This paper is about Poincarés view of the foundations of geometry. According to the established view, which has been inherited from the logical positivists, Poincaré, like Hilbert, held that axioms in geometry are schemata that provide implicit definitions of geometric terms, a view he expresses by stating that the axioms of geometry are "definitions in disguise." I argue that this view does not accord well with Poincarés core commitment in the philosophy of geometry: the view that geometry is the study of groups of operations. In place of the established view I offer a revised view, according to which Poincaré held that axioms in geometry are in fact assertions about invariants of groups. Groups, as forms of the understanding, are prior in conception to the objects of geometry and afford the proper definition of those objects, according to Poincaré. Poincaré's view therefore contrasts sharply with Kant's foundation of geometry in a unique form of sensibility. Accordingly, axioms are not definitions in disguise because they themselves implicitly define their terms, but rather because they disguise the definitions which imply them. According to my interpretation, axioms are not definitions in disguise because they themselves implicitly define their terms, but rather because they disguise the definitions which imply them.
\end{abstract}

Jeremy Shipley

Volunteer State Community College, Gallatin, TN 37066 e-mail: jshipley6@ volstate.edu

This work was supported by funding from the Lorraine Region and much of the research was conducted at the Archives Henri Poincare at the Université de Lorraine. Early versions of the paper were presented to seminars at the Université de Lorraine and Universit at Konstanz in 2013. Subsequent versions were presented at the Midwest Philosophy of Mathematics Workshop in 2015, the Canadian Society for the History and Philosophy of Mathematics Annual Meeting, the Lehigh University Annual Conference in Philosophy, and the Biennial Meeting of the Philosophy of Science Association in 2016. I would like to thank Gerhard Heinzmann, Philippe Nabbonaud, JeanPierre Ferrier, Bruno Blind, Denis LeLarge, Jacintho Del Vecchio, Dina Emundts, Jacob Rosenthal, Jochen Briesen, Amber Griffioen, Gregory Landini, Colin McLarty, and Jamie Tappenden for helpful discussions of the topic of this paper. 


\section{Introduction}

In this paper I will offer an interpretation of Henri Poincaré's views on the foundations of mathematics, revolving around a revised assessment of his understanding of geometry. My interpretation of Poincaré on the foundations of geometry departs from the established view, according to which he held that axioms implicitly define geometric terms. In the following section I will present the established view and briefly summarize the textual evidence for it. Subsequently I will explain and provide evidence for my revised view. According to the established view, Poincaré regarded the axioms of geometry as uninterpreted schemata, providing implicit definitions of geometrical terms. I will contend that Poincaré in fact maintained that the axioms of geometry are properly understood as assertions about invariants of geometric groups.

This paper extends and clarifies the interpretation of Poincaré's philosophy of geometry that has recently been articulated by David Stump and Gerhard Heinzmann. In particular, I disagree with part of Stump and Heinzmann's Stanford Encyclopedia of Philosophy article on Poincaré; viz., the claim that "concerning pure and applied geometry, Poincaré holds the modernist-sounding view that we have no preaxiomatic understanding of geometric primitives" (Heinzmann and Stump, 2014, $\S 3.2) .{ }^{1}$ I will argue that according to Poincaré we do indeed have a pre-axiomatic understanding of geometric primitives. The revised view that I will defend aligns well with Michael Friedman's interpretation of Poincaré's conventionalism, which emphasizes the group theoretical perspective that is key to comprehending Poincaré's comments on geometry (Friedman, 1997). The paper contributes to the literature primarily by directly addressing the established view, articulating an alternative and showing how that alternative is better supported; secondarily, I will clarify the relationship of Poincaré's views to those of his contemporaries by showing that, contrary to Russell's perception, the Poincaré-Russell polemics concerned not whether geometrical primitives should receive nominal definitions but rather what are the primitives, and also by emphasizing the differences between Poincaré and Hilbert.

\section{The established view}

According to to the established view, Poincaré understood geometric axioms to be uninterpreted schemata that define mathematical concepts without reference to intuited objects. Accordingly, many have thought that Poincaré held that geometrical concepts are to be defined by implicit definition. This view of Poincare can be found expressed by the logical positivists, and it influenced their understanding of his conventionalism. Moritz Schlick wrote:

\footnotetext{
${ }^{1}$ My interpretation, however, is strongly influenced by Heinzmann, and in particular on his view of Poincaré's account of mathematical reasoning as founded on the "capability to follow an action schema" (Heinzmann, 1998, p. 47).
} 
To define a concept implicitly is to determine it by means of its relations to other concepts. But to apply such a concept to reality is to choose, out of the infinite wealth of relations in the world, a certain group or complex and to embrace this complex as a unit by designating it with a name. By suitable choice it is always possible under certain circumstances to obtain an unambiguous designation of the real by means of the concept. Conceptual definitions and coordinations that come into being in this fashion we call conventions (using this term in the narrower sense, because in the broader sense, of course, all definitions are agreements). It was Henri Poincaré who introduced the term convention in this narrower sense into natural philosophy; and one of the most important tasks of that discipline is to investigate the nature and meaning of the various conventions found in natural science (Schlick and Blumberg, 1974, p. 71).

Rudolf Carnap, too, mentioned Poincaré's philosophy of science in the context of his discussion of "structural definite descriptions," which he relates to Hilbert's implicit definitions, suggesting that he too viewed Poincaré's conception of geometry as aligned with the idea of implicit definitions (Carnap, 1969, p. 27-30). ${ }^{2}$ According to the established view, convention enters into science through the coordination of axiom schema to reality.

More recently, Stewart Shaprio, in his book defending in re structuralism, situates Poincaré as a proponent of implicit definitions of geometrical concepts by uninterpreted axiom schemata (Shapiro, 2000, p. 154-156). Shapiro draws his interpretation from the influential work of Alberto Coffa, who parallels the Poincaré-Russell polemics on the foundations of geometry with the Hilbert-Frege correspondence, by aligning Poincaré and Hilbert on the satisfactoriness of implicit definitions and Russell and Frege on the demand for nominal definitions (Coffa, 1993). While it is true that Russell understood Poincaré to be rejecting the need for nominal definitions of geometric primitives, on my view this is a misunderstanding on Russell's part: Poincaré was arguing that Russell had the wrong primitives and not that there wasn't any need for primitives. Here is how Coffa presented the basic issue at stake in that debate:

[Russell's] reasoning [against Poincare] involved an appeal to a principle that may be called the thesis of semantic atomism. . This principle says that if a sentence $\mathrm{S}$ is to convey information (or, as Russell or Frege would put it, to express a proposition), then its grammatical units must have a meaning before they join their partners in S (Coffa, 1993, p. 131)...

Poincare's conventionalism was based on the idea that in order to understand geometry, one must stand Russell's argument on its head: Since geometric primitives do not acquire their meaning prior to their incorporation into the axiomatic claims, such axioms do not express propositions in Frege's or Russell's sense (Coffa, 1993, p. 133).

To put a fine point on it, I will show that according to Poincaré the geometric "primitives" in question do in fact acquire meaning prior to their incorporation in typical axiomatizations, precisely because they are not, after all, primitive.

Though I will be offering an alternative, there can be no doubt that there is a textual basis for following the positivists, Coffa, Shapiro, and others in accepting the established view. The established view is supported by three pillars:

\footnotetext{
${ }^{2}$ However, Carnap views Poincaré as not having gone far enough, since, according to Carnap Poincaré conceived of science as concerned with relations rather than taking the further step of saying science is concerned with the Russellian structure-type of a relation.
} 
1. The established view provides an appealing and direct explanation of Poincaré's statement that axioms are definitions in disguise.

2. The established view also explains why Poincaré concurs with Hilbert's claim that mathematics is constrained by consistency alone.

3. Following Schlick, the established view coheres with a common understanding of Poincaré's conventionalism.

Defenders of the established view may lean upon Poincaré's repeated insistence that axioms are definitions in disguise, constrained only by non-contradiction, and that they are conventions. How else can this be understood than in accordance with the established view? Accordingly, axioms are definitions in disguise in the sense that they implicitly define the concepts of point, line, plane, etc. by characterizing a relational structure among terms, which then must be coordinated with the world by appropriate conventions.

In Science and Value Poincaré argues that applied geometry concerns neither synthetic a priori judgments nor experimental facts. It does not concern synthetic a priori judgments because it is possible to imagine experiences that would lead us to deny some of the axioms of Euclidean geometry, and it does not concern experimental facts because it is an exact science. He concludes that the claims of applied geometry are conventional:

They are conventions. Our choice among all possible conventions is guided by experimental facts; but it remains free and is limited only by the necessity of avoiding all contradiction.

Thus it is that the postulates can remain rigorously true even though the experimental laws which have determined their adoption are only approximate.

In other words, the axioms of geometry (I do not speak of those of arithmetic) are merely disguised definitions (Poincaré, 1905, p. 65)

Understood in the following way, this characterization of the axioms of geometry accords with a view commonly associated with Hilbert. Axioms are possible conventions because they are schematic and are to be coordinated to empirical reality by conventional definitions. A "line" may be a rail track or a ray of light, and a "point" may be a rail station or a star. ${ }^{3}$ Using the inferential relations encoded in the axioms, we form abstract mathematical concepts of point, line, etc. as places in an abstract structure, but there is no privileged, intuitive interpretation according to which the truth or falsity of the axioms is to be assessed. Accordingly, axioms provide implicit definitions of general concepts, and are only apparent assertions about objects. ${ }^{4}$

\footnotetext{
${ }^{3}$ Hilbert is credited with having said of geometric terms that "One must be able to say "tables, chairs, beer mugs' each time in place of 'points, lines, places',' (Blumenthal, 1935, p. 402-403).

${ }^{4}$ A great deal has been written about Hilbert's view in his Grundlagen der Geometrie, the relationship between this view and the modern model theoretical understanding of axioms, and Frege's response to Hilbert in their correspondence. A reviewer points out that an appeal to Fregean secondorder concepts can accommodate Hilbert's method, because axioms, understood as implicit definitions, define classes of structures. This proposal is discussed in William Demopolous's essay "Frege, Hilbert, and the Conceptual Structure of Model Theory" (Demopoulos, 1994, p. 219). Patricia Blanchette has argued that Frege, while failing to adequately appreciate Hilbert's method and the new sense of 'consistency' that method involved, does succeed in articulating a sense in which Hilbert fails to demonstrate the consistency of denying the parallel postulate: viz., when that postu-
} 
In the context of the established view, good sense is made of the fact that Poincaré, like Hilbert in his correspondence with Frege, emphasized that consistency was the only constraint on mathematics, stating that "a mathematical entity exists, provided its definition implies no contradiction, either in itself, or with the propositions already accepted" (Poincaré, 1905, p. 60). Such passages may incline one to assimilate Poincaré's views on the foundations of geometry to Hilbert's and to analogize the Hilbert-Frege and Poincaré-Russell polemics. For, the claim that mathematics is constrained only by consistency may seem naturally to be understood in a framework that prioritizes axiomatization over genetic intuitions, because a property naturally ascribed to sets of sentences or propositions, viz. consistency, is given priority over intuition as a criteria of existence.

Lastly, it may also be contended that Poincaré's famous non-falsifiability arguments for geometrical conventionalism are only to be understood in the context of the established view. Any axiom system for geometry can be adopted and held to come what may, making compensating changes to other theories should anomalous observations occur. Accordingly, geometrical axioms must be conventions because of their non-falsifiability in practice. The relational structure encoded in the implicit definition is non-falsifiable for the system to which the definitions are coordinated, and hence it is conventional.

\section{A Revised Interpretation}

Notwithstanding the support available for the established view, I will argue in what follows that it leads us to neglect important distinguishing features of Poincaré's view of the foundations of geometry, that the sense in which axioms are disguised definitions differs for Poincaré from Hilbert's sense, that Poincaré's conventionalism can only be restricted to geometry (rather than implying a more radical DuhemQuine style holism) if the established view is rejected, and that Poincaré's and Hilbert's equal emphasis on consistency is circumstantial.

The central feature of the established view is the treatment of geometrical axioms as schematic rather than assertoric. According to the revised interpretation of Poincaré's views on geometry that I wish to advance, geometric axioms are assertions about points and lines, which are defined prior to axiomatization by what Poincaré calls "invariant" sub-groups (in modern parlance: "normal" sub-groups). This interpretation allows us to see Poincaré's views on the foundations of geometry as intimately related to his mathematical practice and to his emphasis on the importance of group theory. While the established view may be fully articulated without

late is understood as expressing a Fregean thought (Blanchette, 1996). The motivation for Frege's resistance to Hilbert's approach, and to regarding geometry as a branch of logic like arithmetic, has been traced to his experience with 19th century geometry, beginning with (Wilson, 1992) and further developed in (Tappenden, 1995). I discuss Frege's position that geometry is semantically founded on reference to particulars presented in sensible intuition in my "Frege on the Foundation of Geometry in Intuition" (Shipley, 2015). 
mention of group theory, the revised interpretation gives central place to Poincaré's insistence that geometry is the study of the properties of certain groups.

Poincaré's claim that geometry is the study of a group makes its first appearance in his reputation-making mathematical work on Fuchsian functions, produced in 1880. In a supplement to the prize essay submitted to the Académie des Sciences in Paris he states:

It [geometry] is the study of the group of operations formed by the displacements to which one can subject a body without deforming it. In Euclidean geometry the group reduces to the rotations and translations. In the pseudogeometry of Lobachevski it is more complicated (Poincaré, 1997, p. 14).

The primary concern of this work is differential equations rather than geometry and its foundations, and these early comments do not receive full philosophical articulation. Geometry arises in the course of studying and classifying functions that are solutions to second order differential equations having specified properties. ${ }^{5}$ The key to Poincaré's discoveries in the field of Fuchsian functions was his realization that the transformations employed in the definition of Fuchsian functions form a group that is isomorphic to the group employed in the definition of the hyperbolic geometry of Lobachevsky, leading him to the analogy "the Fuchsian function is to the geometry of Lobachevsky what the doubly periodic function is to that of Euclid" (Poincaré, 1997, 15). In a text originally composed in 1901 and later published in Acta Analytica in 1921, Poincaré emphasizes the fundamental role of group theory by asserting an analogy between his work on solutions to classes of differential equations and Galois' work on the solution of polynomials by radicals (Poincaré, 1983, 259). According to the interpretation that I am arguing for, Poincaré's philosophical commitments in the foundations of geometry are intimately related to his formative mathematical experience and to a broader view of mathematics.

Groups play a fundamental role in Poincaré's more philosophical account of the conventions we employ in order to categorize and control the changes that occur in our perceptual stimulus presented in his 1898 article "On the Foundations of Geometry" published in The Monist. Note well that the form of a group of operations that is imposed in categorizing sensible changes as displacements does not, in Poincaré's view, depend in any way on the qualitative character of the sensible changes. It is, rather, strictly formal and it's properties can be studied formally. Poincaré writes:

We have now to study the properties of the group. These properties are purely formal. They are independent of any quality whatever, and in particular of the qualitative character of the phenomena which constitute the change to which we have given the name displacement. We remarked above that we could regard two changes as representing the same displacement, although the phenomena were quite different in qualitative nature. The properties of this displacement remain the same in the two cases; or rather the only ones which concern us, the only ones which are susceptible of being studied, are those in which quality is no wise concerned (Poincaré, 1898, p. 13).

${ }^{5}$ For details see John Stillwell's helpful introduction and the primary texts (Poincaré, 1985) as well as Jeremy Gray's commentary accompanying the further texts (Poincaré, 1997). 
Continuing, Poincaré defines and discusses the notion of isomorphism, and the idea that the formal properties of a group are what are studied by mathematics. For example, the group of permutations of five cards is isomorphic to the group of permutations of five marbles; the qualitative difference between cards and marbles is irrelevant to mathematics. Similarly, all that matters to mathematical geometry are the formal properties of the group of displacements.

The structure of a group affords the definitions of the straight line and of the point given in the Monist article. Poincare arrives at the notions of "point" and "line" in an entirely group theoretically driven way. The rotative subgroup is prior in conception to the point, which is conceived only as that which is fixed by the rotation, and the rotative sheaf, defined by taking the common displacements of two rotative groups, is prior in conception to the line, which is conceived as the axis that is fixed by a rotation: "Here [the with the rotative sheaf] is the origin of the notion of the straight line, as he rotative sub-group was the origin of the notion of the point." (Poincare, 1898, p. 20). Poincaré insists that although the idea of a rotative group is suggested by sensation, it is conceived in a purely formal manner by the understanding, so that our reasoning may become precise. ${ }^{6}$ There is a genuine ambiguity in the text. Poincaré refers to the rotative sub-group and sheaf as, respectively, the "origin" of our notions of point and line. I will not take a position on how to interpret this. On one interpretation the points and lines just are the sub-groups. According to another interpretation, points and lines are parts of an amorphous, continuous manifold, which are invariant under the action of the sub-groups in question. ${ }^{7}$

\section{Definitions in Disguise}

This brings us to the first pillar of support for the established view. What sense can be made of Poincaré's claim that geometrical axioms are "definitions in disguise" if the established view is abandoned? Is there a tension between the view of axioms as definitions in disguise and the definition of points and lines as group invariants? In fact, keeping in mind that Poincaré gives prior and independent definitions of the primitive terms occurring in geometric axioms can help us to better understand what he means when he says that axioms are definitions in disguise, and how his intended meaning differs from the view that has been attributed to him.

For Poincaré the claim that axioms are definitions in disguise does not mean that the axioms are themselves definitions that are disguised as assertions. Rather,

\footnotetext{
${ }^{6}$ This repeats a theme in Poincaré's work, wherein vague, inaccurate, or even inconsistent ideas are suggested by sensation then made precise and consistent through mathematical reflection. His discussion of Fechner's account of the subjective continuum and its precisification in mathematical understanding, for example, follows this pattern (Poincaré, 1905, p. 22).

${ }^{7}$ Because of this ambiguity, and because I take no position on the correct interpretation, I have not commented on the status of Poincaré's analysis situ in this paper. However, there is evidence in his review of Russell's Essay On the Foundations of Geometry that in the 1890s he conceived of analysis situ, like metrical and projective geometry, as founded on a group of transformations (Poincaré, 1899, §19)
} 
axioms are definitions in disguise because they disguise the definitions that imply them. One reason to adopt this interpretation is that it allows us to make the claims about axioms and definitions cohere with the plainly stated definitions of "point" and "line" given in "On the Foundations of Geometry" (the 1898 Monist article) and to make sense of the consistently insisted upon priority of group theory. However, the reasons for adopting this interpretation are not exclusively indirect, and in fact there is strong textual evidence for this way of viewing things. When read in full context it is clear that while Poincare does, of course, say that axioms are definitions in disguise, he does not mean that axioms implicitly define mathematical concepts; rather, he means that there are correct definitions that imply the axioms, which are disguised when we treat the axioms as primitive.

In Science and Hypothesis the claim that axioms are "disguised definitions" comes in the summation of Ch III, and is presented as an alternative way to state that the axioms of geometry "are neither synthetic a priori judgments nor experimental facts," but rather are "conventions" (Poincaré, 1905, p. 65). We shall return to the relevance of the revised interpretation that I am arguing for to the proper understanding of Poincaré's conventionalism shortly, but for now I wish to focus attention on passages which precede these widely cited quotations, which I claim will help us better understand the statement that axioms are definitions in disguise.

In a section of Ch III titled "The Implicit Axioms" Poincare begins with the question "Are the axioms explicitly enunciated in our treatises the sole foundations of geometry?" The answer implied by the ensuing discussion is plainly "no." There are unenunciated "implicit axioms" that are implied by the correct definitions of geometrical entities. It is important to stress that Poincaré holds clearly that there are correct definitions of geometrical entities in this section of Science and Hypothesis and that these definitions, which entail the implicit axioms, are the same as those given in "On the Foundations of Geometry":

"It may happen that the motion of a rigid figure is such that all the points of a line belonging to this figure remain motionless while all the points situated outside of this line move. Such a line will be called a straight line." We have designedly, in this enunciation, separated the definition from the axiom it implies.

Many demonstrations, such as those of the cases of the equality of triangles, of the possibility of dropping a perpendicular from a point to a straight, presume propositions which are not enunciated, for they may require the admission that it is possible to transport a figure in a certain way in space (Poincaré, 1905, p. 62).

Contrary to the view that Poincaré held that the usual axioms provide implicit definitions of geometric concepts, Poincaré is here articulating (less than perfectly clearly, it must be admitted) the same view that he holds in "On the Foundations of Geometry", that we obtain concepts like "point" and "line" as invariant elements of group actions; these are what determine the possibility of transporting figures. He writes that affirming the existence of a certain group of operations "constitutes the axioms of Euclid" (Poincaré, 1898, p. 18). Indeed, I do not think that these passages of Science and Hypothesis can support the claim that Poincaré rejects the traditional demand for nominal definitions. Poincaré is, rather, insisting that the nominal definitions that have traditionally been given are inadequate, and need to be replaced by 
definitions that constitute geometrical objects based on the conventional assumption of a chosen group; this "true definition" is not an implicit definition in the sense of Schlick.

\section{Consistency}

So much for the first pillar of support for the established view. We have seen that the revised interpretation of Poincaré's foundation of geometry that axioms are definitions in disguise can equally well, if not better, account for the relevant texts. Accordingly, axioms are not definitions in disguise because they themselves implicitly define their terms, but rather because they disguise the definitions which imply them. We turn now to the second support for the established view: viz., that it makes the best sense of Poincaré's concurrence with Hilbert that mathematics is constrained only by non-contradiction. To better understand Poincaré's view on this, we should situate his thinking on the foundations of geometry in relation to Kant's.

A clear agenda of Poincaré's Monist article is to vitiate Kant's account of the origin of geometry in a single synthetic a priori form of sensibility and to replace that account with a theory of the origin of geometry in the understanding. Importantly, Poincaré's proposal for doing this conceives of forms of the understanding as groups of operations rather than discursive concepts. That Poincaré opposes the account of geometric notions as originating in sensibility, either empirically or transcendentally, is clear from page 1 of the essay:

Our sensations cannot give us the notion of space. That notion is built up by the mind from elements which pre-exist in it, and external experience is simply the occasion for its exercising this power, or at most a means of determining the best mode of exercising it.

Sensations by themselves have no spatial character (Poincaré, 1898, p. 1).

The remainder of the essay is devoted to articulating an alternative, based on the claim that "we have within us, in a potential form, a certain number of models of groups, and experience merely assists us in discovering which of these models departs least from reality" (Poincaré, 1898, p. 13), which form "the common patrimony of all minds" (Poincaré, 1898, p. 18).

Now, on the established view we can well understand how geometry could be founded in the understanding rather than sensibility. Accordingly, geometry concerns relations between what Kant called "discursive concepts", which are constrained only by the law of non-contradiction but which, according to Kant, as empty linguistic forms thus lack "objective validity." 8 Though it is a bit anachro-

\footnotetext{
${ }^{8}$ Early in the transcendental aesthetic, Kant denies that space is a "discursive concept" (Smith, 1929, B37). According to Kant, the process of geometrical object construction by the productive imagination is governed and restricted by space as a form of sensibility. The formation of concepts in the understanding is restricted only by the law of non-contradiction, but some non-contradictory concepts, such as that of a figure closed by two straight lines, are nevertheless concepts of impossible objects. They lack objective validity. Kant distinguishes between "form of thought", which has a purely logical significance, and the conditions for the possibility and necessity of objects
} 
nistic, I think it is not entirely misleading to assimilate Kant's "discursive concepts" or "forms of thought" to Schlick's implicit definitions. Hilbert's claim that the only constraint on mathematics is the law of non-contradiction, that in mathematics existence means non-contradiction, can be read in this context as rejecting the Kantian standard for objective validity and that any consistent axiomatization may have a corresponding model freely postulated. Recalling that Poincaré makes similar statements to Hilbert's concerning consistency and existence, ought we not, as the second pillar of the established view has it, interpret him in the same way, and ought we to after all attribute to Poincaré a view that gives axiom schemata and implicit definition a central role?

An alternative, non-discursive, interpretation of Poincarés view of the role of non-contradiction is available, which better accords with his insistence on group theoretical foundations of geometry and with algebraic methods that Poincaré celebrated as fundamental to the advance of mathematics in the 19th century. ${ }^{9}$ I wish to distinguish the question of the consistency of an extension of a system of operations from the question of the consistency of a set of propositions. On the one hand, if we understand the claim that mathematics is constrained only by non-contradiction as applying to consistency in the latter sense, then we are lead naturally to Hilbert's view and to the axiomatic method as a mode of definition of mathematical concepts, which may be considered as discursive concepts in Kant's sense; we just drop the constraint of objective validity and its association with transcendental conditions of sensory representation.

Alternatively, we may view consistency as restraining the freedom we have in postulating extensions of groups of operations. Treating consistency as a constraint on extending systems of operations, rather than on the acceptability of axiom schemata as implicit definitions, allows one to understand the consistency constraint in a way that harmonizes well with Poincarés description of the development of number systems beginning with basic arithmetic. Therein, we take for granted an understanding of the operation +1 and use this understanding to define further operations by recursion, through a "monotonous series of reasonings" (Poincaré, 1905, p. 36). It is in this context that Poincaré makes his controversial remarks regarding the non-reducibility of the principle of recursion to the principle of non-contradiction, a claim which he maintained would preclude a complete logicist reduction of mathematics. Our purpose is not to evaluate this claim, however, but to better understand Poincaré's general way of thinking about the foundations of mathematics, and his remarks well accord with the view that he considered consistency to be the constraining factor on the process of extending systems of operations. For it is this

of thought, which concerns experience and its synthetic unity (Smith, 1929, B267). To determine that a concept is of a possible object of thought, it is necessary but not sufficient to show that it is non-contradictory. Sufficiency demands, further, that a concept must satisfy the conditions for the possibility of experience. The point is made nicely, by Michael Friedman (Friedman, 1990, p. 216-218) who references the following passages: (Smith, 1929, B271) and (Smith, 1929, B298-9). See also (Smith, 1929, B151-2) and (Smith, 1929, B196) for more on objective validity and the relationship between concepts formed in the understanding and the forms of sensibility through construction by the productive imagination.

${ }^{9}$ See (Poincaré, 1983, 259), which is discussed above. 
constraint that we know to be unviolated by "the power of the mind which knows itself capable of conceiving the indefinite repetition of the same act when once this act is possible" (Poincaré, 1905, p. 39).

We may bring this discussion of Poincaré's view of the foundations of arithmetic into connection with his discussion of the operations that form the groups at the foundation of geometry by reflecting on the following passage, which introduces the section of "On the Foundations of Geometry" that is concerned with rotative sub-groups:

The number of sub-groups [of the group of all displacements] is infinite; but they may be divided into a rather limited number of classes of which I do not wish to give here a complete enumeration. But these sub-groups are not all perceived with the same facility. Some among them have been only recently discovered. Their existence is not an intuitive truth. Unquestionably it can be deduced from the fundamental properties of the group, from properties which are known to every body, and which are, so to speak, the common patrimony of all minds. Unquestionably it is contained there in germ; yet those who have demonstrated their existence have justly felt that they had made a discovery and have frequently been obliged to write long memoirs to reach their results.

Other sub-groups, on the contrary, are known to us in much more immediate manner. Without much reflexion every one believes he has a direct intuition of them, and the affirmation of their existence constitutes the axioms of Euclid. Why is it that some sub-groups have directly attracted attention, whilst others have eluded all research for a much longer time? We shall explain it by a few examples (Poincaré, 1898).

There are two ideas to draw from this passage that speak in favor of the revised interpretation. First, in general support of the revised interpretation, the conceptual priority of groups of operations is affirmed by the claim that the axioms of Euclid are constituted by the existence of a group. Second, in support of the present reflections on the consistency constraint, the passage strongly suggests the foundational importance of research into groups and their subgroup structure, which I would suggest is best understood on the model of Poincaré's view of the foundations of arithmetic in the following way: a "germ" is developed through the repetition and combination of generating operations ${ }^{10}$ I elaborate on this idea, and the relationship the way groups may be defined and some of Poincaré's broader commitments regarding proper definition below.

In the light of the role that the consistency constraint has for Poincare that I have been developing, we can see how consistency can have been important for him without relying on the established view that he took axioms to be schemata and to function as implicit definitions. Specifically, consistency constrains the construction of forms of the understanding by restricting the introduction of generators or relations either primitively or to extend existing groups.

\footnotetext{
${ }^{10}$ Compare the "monotonous series of reasonings" characteristic of arithmetic and the "long memoirs" on the classification of groups. Poincaré's impatience with this sort of work, as an "intuitive mathematician", should not be read as a dismissal of its foundational importance. See (McLarty, 1997) for more on Poincaré's view of rigor.
} 


\section{Conventionalism}

So much for the second pillar supporting the established view. I turn now to the third pillar: that the established view lends to a nice account of Poincaré's conventionalism. Schlick's interpretation of Poincaré, as we have detailed above, makes natural sense of his conventions in the following way: conventions coordinate terms in implicit definitions to real objects, properties, and relations. The main problem for the established view concerning the interpretation of Poincaré's conventionalism is that it leads to a difficulty in saying why we should be, as Poincaré held, conventionalists about geometry in particular, rather than about other sciences. For, if our conventionalism derives from that fact that once terms occurring in axiom schemata are coordinated with reality, the schemata may be held to come what may then the same can be done for fields besides geometry, such as kinematics.

As Michael Friedman has argued, only an appeal to the priority of group theory in Poincaré's hierarchy of sciences can make sense of why he was a conventionalist about spatial geometry and not kinematics:

Now Poincaré's conception of geometry is also very similar to the Kantian conception of geometry. For Poincaré, as for Kant, geometry is synthetic, because it is based, like arithmetic, on the possibility of indefinitely repeating particular operations: namely, group-theoretical operations constituting a Lie group of free motions. Moreover, geometry is viewed as the presupposition of all empirical physical theories: neither for Poincaré nor for Kant can geometry itself be either empirically confirmed or empirically disconfirmed. The difference, of course, is that Poincaré, in contrast to Kant, is acquainted with alternative geometries. Poincare is acquainted, in particular, with the Helmholtz-Lie theorem, according to which geometry is constrained, but by no means uniquely determined, by the idea of a Lie group of free motions. It then follows for Poincaré because three alternative possibilities are still left open, that we have here-in this very special situation-a conventional choice or free stipulation (Friedman, 1997, p. 312).

There is a long story to be told about the relationship between Poincaré's hierarchical view of science and his restricted conventionalist thesis. A full telling is beyond the scope of the present discussion, but it suffices to concur with Friedman that the revised interpretation is better situated than the established view to make sense of why Poincaré's conventionalism is restricted to geometry and does not tend toward more radical versions of confirmational holism based on the contention that any proposition may be held onto in the face of apparently contradictory evidence, as endorsed for example by Quine (Quine, 1951). According to the established view of Poincaré's conventionalism, geometry is conventional because it is non-falsifiable. To block falsification one simply handles anomalies by altering the kinematics. However, the relationship between geometry and kinematics is symmetrical on this account. Should we choose to stubbornly adopt a theory in kinematics, we could resist falsification by making compensating changes to our geometry. On the revised interpretation, geometry is non-falsifiable because it is conventional, a choice of a group must be made before empirical science can proceed. 


\section{Defining Groups}

I have argued that, as with arithmetic, Poincaré understood groups to be generated by potentially indefinite iteration of a generating element. In the case of discrete groups, this may be done by specifying generators and relations. In the case of continuous groups, we need infinitesimal generators. It may be objected that this approach, after all, leans on implicit definitions, given the formalist character of group presentations. However, even if it is granted that group theory in general rests on implicit definition, the definitions of the terms contained in the typical axioms of geometry would not be implicitly defined. In fact, there is good reason to think that Poincaré would want implicit definition to be restricted to the algebraic setting, given his comments on definition in his later works. In an essay contained in the collection Dernières Pensées, Poincaré distinguishes two cases of what he calls "definition by postulate", which may plausibly be considered to be implicit definitions. The case which is objectionable to the "pragmatist" and accepted by the "Cantorian" occurs when an object is defined implicitly by a postulate which relates it to an infinity of elements in a supposedly given domain. However, this form of definition is acceptable to the pragmatist when an element is defined by its relation to objects in a finite domain (Poincaré, 1963, p. 69-71). Katherine Dunlop has pointed to these passages to argue that Poincaré cannot succeed in founding geometry on group theory in a way that does not run afoul of his restrictions on definition, because "a group is characterized by the existence of an identity element that bears a specified relation to every object in the group" (Dunlop, 2016, p. 303). However, when a group is presented by a finite set of generators and their relations, only the relation of the generators to the identity must be stipulated, and the universal generalization to all elements can be proven by an inductive argument. On the other hand, axioms of geometry, taken as implicit definitions, would run afoul of this constraint on definitions (and worse); for, they aim to simultaneously define infinitely many points and lines by simultaneously stipulating relations to other objects in the same infinite set.

Furthermore, I would contend that Poincaré's account of indefinite repetition and continuity, both of which will be involved in constructions from infinitesimal generators, appeal only to a kind of intellectual intuition, and not, as Janet Folina has argued, to Kantian forms of sensible intuition (Folina, 1992, p. 35-36, p. 134-135, p. 180-181). Poincaré's views on these matters are, however, subtle and possibly shifted in his later texts in ways that are favorable to Folina's reading. A competent and complete engagement with Folina's thorough treatment of Poincaré's views is beyond our present scope. If Folina's view is correct then there is an ineliminable appeal to forms of sensibility that is required to adequately define the groups and sub-groups that we have been discussing. While this undermines the position that Poincaré rejects forms of sensibility and founds mathematics entirely on forms of the understanding, which is the strongest view I am inclined to endorse, it leaves untouched my core contention that group theory is prior in conception to to the axioms of geometry. 


\section{Poincaré and His Contemporaries}

A recurring theme of this paper has been the comparison of Poincaré's and Hilbert's images of mathematics. According to the established view there is little difference between the two on geometry, with the primary difference comes in Poincaré's proto-intuitionist view of arithmetic and the irreducibility of arithmetic induction to logic. ${ }^{11}$ According to the view espoused in this paper, Poincaré held that group theory was the foundation of both arithmetic and geometry. Arithmetic concerns the important special case: the infinite cyclic group, otherwise known as the integers. The groups that constitute the forms of the understanding, however, include continuous groups with the invariant subgroups that constitute the points and lines about which axioms assert truths. In each case, the construction of these forms occurs through the mind's power to adjoin and combine operations.

Poincaré, to be sure, well appreciated the power of the model theoretic methods employed by Hilbert in his Grundlagen der Geometrie, and praised them in his review. Poincaré especially praised the usefulness of Hilbert's work for breaking down the bias of geometers toward thinking that there is a "general geometry", with Euclidian, Lobachevskian, and Riemannian geometry as special cases. Hilbert's early model theoretic approach effectively demonstrated that it was not only the parallel postulate that could be regarded as logically independent:

But why, among all the axioms of geometry, should [the parallel postulate] be the only one which could be denied without offense to logic? Whence should it derive this privilege? There seems to be no good reason for this, and many other conceptions are possible (Poincaré, 1903, p. 2).

In particular, Hilbert's approach to independence proofs demonstrates the existence of non-Pascalian and non-Desarguesian geometries. Throughout the review, Poincaré demonstrates a clear understanding of Hilbert's methods and an admiration for the results obtained, but does this indicate agreement with the view that the supposedly basic terms of geometry are defined contextually by implicit definitions?

I think that the answer is clearly "no", and this is shown by Poincaré's concluding remarks from the review. There, Poincaré again emphasizes the group theoretical standpoint. First, he notes that, according to Hilbert, "The objects which he calls points, straight lines, or planes become thus purely logical entities which it is impossible to represent to ourselves," but he does not endorse this view (Poincaré, 1903, 22). Rather, he criticizes Hilbert for neglecting the group theoretic definition of these terms, and in particular for failing to note that the non-Pascalian geometries that Hilbert studies may be generated from geometric groups in a way that is analogous to the generation of metrical geometries. The passage culminates in the following criticism:

Professor Hilbert seems rather to slur over these inter-relations; I do not know why. The logical point of view alone appears to interest him. Being given a sequence of propositions, he finds that all follow logically from the first. With the foundation of this first proposition,

${ }^{11}$ A nice discussion of the relationship between Poincaré and full-fledged Brouwerian intuitionism can be found in Folina's book Poincaré and the Philosophy of Mathematics (Folina, 1992, p. 73-74) 
with its psychological origin, he does not concern himself. And even if we have, for example, three propositions A, B, C, and if it is logically possible, by starting with any one among them, to deduce the other two from it, it will be immaterial to him whether we regard $\mathrm{A}$ as an axiom, and derive $\mathrm{B}$ and $\mathrm{C}$ from it, or whether, on the contrary, we regard $\mathrm{C}$ as an axiom, and derive $\mathrm{A}$ and $\mathrm{B}$ from it. The axioms are postulated; we do not know where they come from; it is then as easy to postulate A as C (Poincaré, 1903, p. 22)

If Poincaré held the view that in geometry axioms are arbitrary postulates that implicitly define geometric terms, then this criticism would make no sense. The criticism only makes sense if, as I have argued, Poincaré holds that there is a correct account of where the axioms come from, and this account, as should by now be manifestly evident, requires that the terms contained in the axioms of geometry have proper definitions. According to Poincaré there are non-logical criteria for the correct ordering of propositions in mathematics.

While the Poincaré-Russell polemics have been commonly interpreted as paralleling the Hilbert-Frege correspondence, the orthogonality between Poincaré and Hilbert is in fact previsioned in his review of Russell. Coffa in particular, regards Poincaré as having rejected the thesis of "semantic atomism", according to which the terms contained in a sentence must have prior meanings that must be properly combined for the sentence to express a proposition (Coffa, 1993, p. 131). If this is right, then there is indeed a parallel between Frege's insistence that the axioms of geometry must be assertions about objects that are given in intuition and Russell's supposed semantic atomism. ${ }^{12}$ However, if Poincaré is rejecting semantic atomism then there cannot be correct definitions of the terms "point" and "line", as the 1898 Monist article articulates, that imply the axioms, as described in Science and Hypothesis. ${ }^{13}$

In fact, Poincaré's agenda in Des Fondaments de Geometrie, the review of Russell's 1897 book An Essay on the Foundations of Geometry, is entirely consonant with his agenda in his review of Hilbert. Firstly, he wishes to disavow Russell of the view that there is a core geometry, based on the axioms of projective geometry, that is grounded in sensible intuition. This is precisely the sort of view that Poincaré praises Hilbert for disproving by demonstrating the logical possibility of non-Pascalian and non-Desarguian geometries through model theoretical methods (though, again, Poincaré thought these methods were incomplete because they did not show the origin of these geometries in group theory). Russell's contention, in the 1890 s, was that projective geometry rested on a Kantian foundation in sensible intuition and consisted of synthetic a priori truths, but that metrical geometry was empirical.

It is true that Poincare savagely criticizes Russell's position that the basic terms of projective geometry refer to objects given in sensible intuition, by pointing out

\footnotetext{
12 I think that there are complications with the interpretation of Russell's views in the 1890s implicit in Coffa's commentary, but I limit myself here to correcting the record on Poincaré.

${ }^{13}$ See my discussion above in the section titled "Definitions in Disguise". Notably, the 1898 article was written prior to the publication in 1899 of Poincaré's review of Russell's book on the foundations of geometry. Poincaré's view of geometry as founded in groups theoretical forms of the understanding was motivated by his early mathematical work and held consistently through his writings.
} 
the vagueness and imprecision of Russell's definitions and axioms (Poincaré, 1899, $\S 2-4)$, but this only shows that Poincaré rejected the foundation of geometry in sensibility. As I have argued, his alternative was not a foundation of geometry in discursive concepts defined implicitly by the axioms in which they occur, but rather in group theory as a form of the understanding. Indeed, the group theoretical perspective is what Poincare insists on, and not the rejection of semantic atomism, in presenting his alternative to Russell (Poincaré, 1899, §19). Poincaré wants to disavow Russell of the view he praises Hilbert for dislodging, that there is a universal geometry (for Russell, projective geometry), but he wants to do so by convincing Russell of the priority in conception of the form of the group, which he criticizes Hilbert for neglecting.

There is a clear affinity between Poincaré's view and that of Klein and Lie, expressed in the Erlangen program. Indeed, there was interaction between Poincaré and Lie in 1882 when Lie was in Paris. Lie wrote to Klein that Poincaré held the concept of a group to be the fundamental concept for all of mathematics (Hawkins, 2000 , p. 182). Jeremy Gray notes that it is very likely that Poincaré's use of groups in his analysis of Fuchsian functions was independent of Klein's Erlangen program (Gray, 2005, p. 551). It is not clear whether Klein or Lie ever indicated anything like Poincaré's philosophical view that the group is prior in conception to the terms occurring in the axioms of geometry. Poincaré's position is that group theory defines those terms, that the objects to which they refer are constituted by invariant subgroups, but an alternative view would be that the objects of geometry are presented or constructed independently while groups afford a complete means of classification without necessarily constituting the objects that comprise the spaces thus classified. This view is consistent with a mathematical interest in the use of groups to classify geometries, but inconsistent with Poincaré's fully developed philosophical position.

\section{Conclusion}

This paper has been focused on correctly articulating Poincaré's views on the foundations of geometry. Accordingly, he held that geometry is founded on group theory, in such a way that the terms "point" and "line" receive proper definitions by reference to invariant subgroups. This interpretation sheds light on Poincaré's general philosophy of mathematics. For, group theory, as the study of forms of the understanding, has the same foundation as arithmetic. While Poincaré has sometimes been read as having a disjointed view of mathematics, a kind of quasi-logicist view of geometry as grounded in axiomatic implicit definitions together with a quasiintuitionistic view of arithmetic, we can now see that there is a deep unity in his vision of mathematics.

Should this matter to contemporary philosophers of mathematics, or is getting Poincaré's view right a matter of historical curiosity? I think that, for good reason, philosophers have been fascinated with the notion of mathematics as a science of structure, or logical form, which can be associated with the concepts that are artic- 
ulated through axiomatic definitions. This has given rise to a very interesting literature in the mataphysics of mathematics, on whether structures must be understood as ante rem or in re, what the relationship between structures and sets might be, and many more questions. So it may seem that revisiting a neo-Kantian view from the turn of the 20th century holds limited interest. From this perspective, one may even find that my interpretation of Poincaré in fact diminishes his legacy as anticipating the structuralist turn, based on the axiomatic method, in his writings of the 1890s.

However, while the importance of the axiomatic point of view to the development of mathematics in the 20th century is undeniable, and the understanding of mathematics as advancing through the logical derivation of theorems from axioms, that is as a system of discursive reasoning, certainly has great philosophical importance, the focus on discursive reasoning limits our perspective on the nature of mathematical reasoning, on mathematical experience, and potentially on the very nature of the mind and intelligence. Poincarés unified image of mathematics, derived from his own mathematical experience, beginning with his work on Fuchsian functions, which produced germinal contributions to mathematical knowledge, begins with an account of non-discursive reasoning. Mathematicians are masters of more than the rules of logic, and a complete account of mathematics will involve accounting for both discursive and non-discursive reasoning, as well as the relationship between the two. Correctly understood, Poincaré's account of the foundations of geometry offers a neglected contribution to this philosophical project.

\section{References}

P. A. Blanchette. Frege and Hilbert on consistency. Journal of Philosophy, 93(7): 317-336, 1996.

O. Blumenthal. Lebensgeschichte. In David Hilbert: Gesammelte Abhandlungen, volume III, pages 388-429. Springer, 1935.

R. Carnap. The Logical Structure of the World: And, Pseudoproblems in Philosophy. Open Court classics. Open Court, 1969.

J. Coffa. The Semantic Tradition from Kant to Carnap: To the Vienna Station. Cambridge University Press, 1993.

W. Demopoulos. Frege, Hilbert, and the conceptual structure of model theory. History and Philosophy of Logic, pages 211-255, 1994.

K. Dunlop. Poincaré on the foundations of arithmetic and geometry. part 1: Against "dependence-hierarchy" interpretations. Hopos: The Journal of the International Society for the History of Philosophy of Science, 6(2):274-308, 2016.

J. Folina. Poincaré and the Philosophy of Mathematics. St. Martin's Press, 1992.

M. Friedman. Kant on the mathematical sciences. Synthese, 84:213-257, 1990.

M. Friedman. Poincaré's conventionalism and the logical positivists. In Machine Discovery, pages 299-314. Springer Netherlands, 1997. 
J. Gray. Felix Klein's erlangen program, 'comparative considerations of recent geometrical researches'. In I. Grattan-Guinness, editor, Landmark Writings in Western Mathematics 1640-1940. Elsevier Science, 2005.

T. Hawkins. The Emergence of the Theory of Lie Groups: An Essay in the History of Mathematics, 1869-1926. Sources and Studies in the History of Mathematics and Physical Sciences. Springer Verlag, 2000.

G. Heinzmann. Poincaré on understanding mathematics. Philosophia Scientiae, 3 (2):43-60, 1998.

G. Heinzmann and D. Stump. Henri Poincaré. In E. N. Zalta, editor, The Stanford Encyclopedia of Philosophy. Spring 2014 edition, 2014.

C. McLarty. Poincaré: Mathematics \& Logic \& Intuition. Philosophia Mathematica, 5:97-115, 1997.

H. Poincaré. On the foundations of geometry. The Monist, 9(1):1-43, 1898.

H. Poincaré. Des fondements de la geométrie. Reveu de Métaphysique et de Morale, 7:251-279, 1899.

H. Poincaré. Poincaré's review of Hilbert's "foundations of geometry.". Bulletin of the American Mathematical Society, 10(1):1-23, 1903.

H. Poincaré. Science and Hypothesis. The Walter Scott Publishing Co., 1905.

H. Poincaré. Mathematics and Science: Last Essays. Dover Publications, 1963.

H. Poincaré. Analyse de ses travaux scientifiques. Proceedings of Symposia in Pure Mathematics, 39(2):257-357, 1983.

H. Poincaré. Papers on Fuchsian Functions. Springer-Verlag, 1985.

H. Poincaré. Three Supplements on Fuchsian Functions. Akademie-Verlag, 1997.

W. Quine. Two dogmas of empiricism. Philosophical Review, 60(1):20-43, 1951.

M. Schlick and A. Blumberg. General Theory of Knowledge. Library of Exact Philosophy. Open Court Pub., 1974.

S. Shapiro. Philosophy of Mathematics: Structure and Ontology. Oxford University Press, 2000.

J. Shipley. Frege on the foundation of geometry in intuition. Journal for the History of Analytical Philosophy, 3(6), 2015.

N. K. Smith. Immanuel Kant's Critique of Pure Reason. Macmillan, 1929.

J. Tappenden. Geometry and generality in frege's philosophy of arithmetic. Synthese, 102(3):319-361, 1995.

M. Wilson. Frege: The royal road from geometry. Noûs, 26(2):149-180, 1992. 